\title{
Los Estados Unidos: Un obstácullo histórico y sinuoso en la evolución de la integración centroamericana
}

\section{The United States: A historic and sinuous obstacle in the evolution of Central American integration}

\author{
Navas Calderón, Benito; Martínez Avendaño, Jairo Terencio; Chiong \\ Alegría, Allan Gustavo; Editor Academico Dr. Angel Sol Sanchez
}

\author{
Benito Navas Calderón \\ josenavas19@yahoo.es \\ Centro de Investigación Para Estudios de Integración \\ (CIPEI) UNAN - León. Maestría en procesos de \\ integración segunda edición. , Nicaragua \\ (iD) Jairo Terencio Martínez Avendaño \\ jairomar@gmail.co \\ Universidad Nacional Autónoma de Nicaragua, \\ UNAN - León. Facultad de Ciencias Económicas y \\ Empresariales. Departamento de Economía., Nicaragua \\ Allan Gustavo Chiong Alegría \\ allanchiong1@hotmail.com \\ Universidad Nacional Autónoma de Nicaragua, \\ UNAN - León. Facultad de Ciencias Económicas \\ y Empresariales. Departamento de Empresariales, \\ Nicaragua \\ Editor Academico Dr. Angel Sol Sanchez \\ Colegio de Postgraduados Mexico, México
}

Revista Iberoamericana de Bioeconomía y Cambio

Climático

Universidad Nacional Autónoma de Nicaragua, León, Nicaragua ISSN-e: $2410-7980$

Periodicidad: Semestral

vol. 2, núm. 4, 2016

czuniga@ct.unanleon.edu.ni

Recepción: 17 Diciembre 2016

Aprobación: 17 Diciembre 2017

URL: http://portal.amelica.org/ameli/journal/394/3941751006/

DOI: https://doi.org/10.5377/ribcc.v2i4.5929

Autor de correspondencia: josenavas19@yahoo.es
Resumen: La unidad centroamericana siempre ha sido el anhelo de algunos Estados y líderes de la región, incluso algunos han ofrendado sus valiosas vidas para hoy formar parte de la reciente historia independiente de las repúblicas. Pero lo fascinante es que las diferentes intervenciones imperialistas han hecho posible que los mismos centroamericanos busquen la solución a sus problemas, de la paz y la unidad de la región, el desarrollo y el crecimiento económico.

Un ejemplo valioso fue la firma de la Carta de San Salvador que crea la Organización de Estados Centroamericanos (ODECA) y que es la base para la firma del Protocolo de Tegucigalpa que crea el Sistema de la Integración Centroamericana (SICA); prácticamente con esta iniciativa empieza a funcionar la primera organización regional en Centroamérica después que se rompió la República Federal Centroamericana.

La unidad centroamericana siempre ha sido el anhelo de algunos Estados y líderes de la región, incluso algunos han ofrendado sus valiosas vidas para hoy formar parte de la reciente historia independiente de las repúblicas. Pero lo fascinante es que las diferentes intervenciones imperialistas han hecho posible que los mismos centroamericanos busquen la solución a sus problemas, de la paz y la unidad de la región, el desarrollo y el crecimiento económico. Un ejemplo valioso fue la firma de la Carta de San Salvador que crea la Organización de Estados Centroamericanos (ODECA) y que es la base para la firma del Protocolo de Tegucigalpa que crea el Sistema de la Integración Centroamericana (SICA); prácticamente con esta iniciativa empieza a funcionar la primera organización regional en Centroamérica después que se rompió la República Federal Centroamericana. Las diferentes justificaciones para intervenir en la región especialmente en Nicaragua por parte de los Estados Unidos de Norteamérica son múltiples y con variadas incoherencias. Los Estados Unidos a partir del 9/11 han expresado su deseo por una sociedad política moderna, es decir, libre de caudillos; manifestando una clara oposición e interviniendo en algunas ocasiones el modelo de Gobierno de Reconciliación y Unidad Nacional impulsado por el presidente Daniel Ortega Saavedra.

Palabras clave: intervención, política exterior, integración regional, Estado - nación. 


\begin{abstract}
Central American unity has always been the desire of some States and leaders of the region, some have even offered their valuable lives to today be part of the recent independent history of the republics. But what is fascinating is that the various imperialist interventions have made it possible for the Central Americans themselves to seek solutions to their problems, peace and unity in the region, development and economic growth. A valuable example was the signing of the San Salvador Charter that creates the Organization of Central American States (ODECA) and that is the basis for the signing of the Tegucigalpa Protocol that creates the Central American Integration System (SICA); Practically with this initiative, the first regional organization in Central America begins to function after the Central American Federal Republic was broken up. The different justifications for intervening in the region, especially in Nicaragua, by the United States of America are multiple and with various inconsistencies. Since 9/11, the United States has expressed its desire for a modern political society, that is, free of warlords; manifesting a clear opposition and intervening on some occasions the model of Government of Reconciliation and National Unity promoted by President Daniel Ortega Saavedra.
\end{abstract}

Keywords: intervention, foreign policy, Regional integration, Nation -State.

\title{
I. INTRODUCCIÓN.
}

El objeto del presente trabajo es exponer los principales eventos históricos producto de la Política Externa de los Estados Unidos de Norteamérica en los países de la región centroamericana, como intereses egoístas disfrazados de principios idealistas, haciendo especial énfasis a la relación jurídica con Nicaragua. Para ello hemos elaborado un hilo documental de forma cronológica respondiendo a un solo modelo de la política exterior norteamericana, el modelo de "Divino Elegido" de los Estados Unidos. La argumentación histórica se ha llevado a cabo recurriendo principalmente a fuentes de investigación de reconocido prestigio. Por otra parte se ha completado la información con artículos específicos para temas concretos como el caso del Sistema de la Integración Centroamericana (SICA).

Ahora trataremos de narrar una reducida cronología compuesta por dos etapas históricas incorporando fechas, nombres y a nuestro sencillo parecer cómo han interferido Los Estados Unidos en la región. Los Estados Unidos han mantenido siempre a lo largo de la historia centroamericana una intervención periódica y sistemática, económica, política y militar especialmente en Nicaragua, continuando con su deseo de incrementar su poder y así poder satisfacer sus intereses de seguridad nacional y prosperidad.

\section{ETAPA - 1: FUNDAMENTOS HISTÓRICOS DE LA POLÍTICA EXTERIOR NORTEAMERICANA.}

\subsection{BASE FILOSÓFICA.}

NotAS DE AUTOR 
Los crecientes escándalos de corrupción de la Iglesia católica romana y los enfrentamientos violentos contra el movimiento protestante liderados en las principales ciudades europeas, provocaron la inmigración, e invasión de un pequeño pueblo europeo hacia nuevas tierras americanas, instalando pequeñas colonias compuesta por irlandeses y británicos. Pero este pueblo traía consigo una fuerte creencia arraigada en su seno y es que representaban al pueblo que interpretó correctamente las sagradas escrituras y por lo tanto hacen la voluntad de Dios.

En un estudio sobre las bases de la política exterior de los Estados Unidos a lo largo de la historia se determinó, que para poder comprender el funcionamiento de la política exterior de los Estados Unidos a través del tiempo, incluyendo a la de hoy en día; hay que comenzar con lo fundamental el carácter del ciudadano estadounidense, los estadounidenses tienen una conducta muy peculiar que los diferencia del resto de los habitantes del mundo: se caracterizan por ser autosuficientes, ambiciosos, innovadores, trabajadores y sobre todo muy individualistas (Cincotta, 1994).

La nueva nación norteamericana tenía como habitantes a individuos, como ya lo habíamos mencionado, con deseos de autosuperación y bonanza personal, si recordamos estos fines concuerdan con el deseo del hombre por satisfacer sus propios deseos que postula "la teoría realista"; sin embargo, en la conducta de los ciudadanos de los Estados Unidos nos topamos con un factor que no encontramos en el realismo: la creencia de estar bendecidos y protegidos por una fuerza superior.

\subsection{BASE JURÍDICA.}

De acuerdo a Cincotta las colonias americanas rompen relaciones con su “Madre Patria” en el año de 1774. Después de casi diez años de guerra el 03 de septiembre de 1783 se firmó el Tratado de París, el cual ponía fin a la guerra y le otorgaba de manera oficial la independencia a las "Trece Colonias".

En los primeros años de los Estados Unidos como nación, adoptó una política exterior aislacionista y de esta manera se mantuvieron tranquilos para desarrollar su proyecto de democracia. Este factor les dio a los norteamericanos una gran ventaja sobre las potencias europeas. La joven nación norteamericana se convenció de que su misión en el mundo (como país superior) consistía en difundir la justicia y la moral, con el fin de fomentar una paz genuina y mundial. Los líderes estadounidenses encontraron en la Doctrina Monroe (1823) y en el Destino Manifiesto (1845) la solución para esta contradicción entre los intereses y los ideales.

\subsubsection{LA DOCTRINA MONROE.}

La Doctrina Monroe (1823), que decreta la prohibición para cualquier nación europea de establecer nuevas colonias en el Continente Americano, en otras palabras, Europa no debía inmiscuirse en los asuntos de América (McDougall, 1997).

\subsubsection{EL DESTINO MANIFIESTO.}

El Destino Manifiesto (1845), que postula que es un deber sagrado de los Estados Unidos expandir su territorio hacia todo el norte de América, debe controlar por completo el hemisferio oeste y continuar con la propagación mundial de la moral y la justicia (Stephanson, 1996). En su proceso de expansión los Estados Unidos adquirieron tierras que le pertenecían a Inglaterra, España, Francia y México. Para el año 1890 ya no quedaban fronteras en tierra firme por obtener, ya habían conquistado la mayor parte de América del Norte. Su territorio nacional era mayor a cualquier nación europea con excepción de Rusia y a todo esto no hay que olvidar que las políticas imperialistas se llevaron a cabo en nombre de Dios (Weeks, 1997).

\section{EFECTOS DE LA POLÍTICA EXTERIOR NORTEAMERICANA APLICADA A CENTROAMERICA (1823 - 1890). 3.1. REACCIONES CENTROAMERICANAS.}

En un estudio sobre las etapas en la historia de la política exterior de los Estados Unidos se determinó que el expansionismo inicia en 1823 con el deseo estadounidense de ejercer sobre el mundo un papel de guía para la humanidad. Esta etapa presenta a una nación con gran poder político y económico - alcanzado gracias a la etapa aislacionista - sin olvidar por supuesto, el compromiso con los valores nacionales y 
el concepto de "Divina Providencia" (Díaz, 2013). A partir de ese momento la región latinoamericana y centroamericana en particular experimentaran los siniestros efectos de los principales pilares de la política exterior estadounidense.

De acuerdo a Cañas Dinarte, para cuando llega la mañana sabatina del 15 de septiembre de 1821, las provincias coloniales españolas de la Capitanía General de Guatemala o Reino de Guatemala como gustaba autollamarse, habían vivido ya una intensa década a favor de la emancipación política de la Corona Imperial Ibérica (Vásquez, 2011).

Tras el fin de la anexión al Imperio mexicano del septentrión decretada en la ciudad de Guatemala el 05 de enero de 1822 y con el apoyo de las provincias sureñas de Nicaragua y Costa Rica y, la instalación del primer Congreso Constituyente se crea la República Federal Centroamericana en junio de 1823. Finalmente se tomó la decisión de que la región se declarara independiente de España, México y cualquier otra potencia del mundo el 01 de julio de 1823 y se apostó por la implementación del régimen federal al estilo estadounidense (Vásquez, 2011).

\subsubsection{CONFLICTO DE INTERESES ENTRE LA POBLACIÓN CENTROAMERICANA EN LA DISTRIBUCIÓN DEL PODER POLÍTICO Y ECONÓMICO}

Mientras tanto Cañas Dinarte, afirma que el desarrollo federativo de la naciente República Federal Centroamericana se vio viciado de entrada por fraudes electorales, indisposición de los Estados a someterse a las decisiones federales, inexistencia de un sistema de recaudación tributaria y sobre todo por el creciente poder de unas facciones en contra de otras.

En realidad fue un nacimiento común, pero dividido al mismo tiempo, tanto en participación efectiva de los representantes de todas las provincias e intendencias como en intereses propios de los diversos grupos.

No paso mucho tiempo para que esos intereses y facciones se manifestaran de lleno en suelo centroamericano para disputar su preeminencia unas con otras, hasta incluso llegar a alianzas militares de unos territorios y Estados en contra de otros.

\subsubsection{CREACIÓN DE LOS ESTADOS NACIONALES CENTROAMERICANOS.}

El fin de los enfrentamientos bélicos demasiado seguidos en la región y la existencia de férreos regímenes, hicieron posible la consolidación de los Estados Nacionales, pero a la vez impidieron acercamientos entre los mandatarios centroamericanos y alejaron cada vez las posibilidades de construcción de un sistema auténticamente federal para las nacientes Repúblicas. De acuerdo a Villalta Vizcarra, esta primera República Federal Centroamericana ha sido el máximo ejemplo de integración política porque llegamos a tener una Constitución General para Centroamérica el 22 de noviembre de 1824 (Vizcarra, 2007).

Mientras tanto según Vizcarra (2007), el rompimiento de la primera República Federal Centroamericana ocurrido en 1838, las repúblicas centroamericanas de Guatemala, Honduras, El Salvador, Costa Rica y Nicaragua siguieron siendo un complejo regional con características muy especiales, vinculadas estrechamente en lo social, en lo económico y en lo cultural, como ya lo estaban geográficamente.

\subsection{LA POLÍTICA EXTERIOR NORTEAMERICANA Y LOS TRATADOS CANALEROS EN CENTROAMERICA.}

Los estadounidenses aprovecharon esta oportunidad de uniones y desuniones para desempeñar su papel injerencista y expansionista en territorio centroamericano y caribeño. Estados Unidos tenía planes de construir un canal en el istmo de Centroamérica con el fin de unir el océano Pacífico con el Atlántico. Lo cierto es que durante cuatro siglos la construcción del canal interoceánico, ha sido la aspiración casi compulsiva e ineludible de gobernantes y gobernados de Nicaragua. Alrededor de este tema se tejieron intrigas, intervenciones, tratados, misiones o meras fantasías.

Según Álvarez Montalván, todo inicia con la búsqueda quimérica del estrecho dudoso que conectaría el Atlántico con el Pacífico, empeño en el cual participaron desde los conquistadores españoles que buscaban ansiosos el paso a nivel, hasta un Emperador de Francia jugando al ingeniero hidráulico, pasando por los 
propios reyes ibéricos y algunos funcionarios estadounidenses que le dieron su nombre a un discutidísimo tratado.

La propia Corona Inglesa, sin dejar a un lado el atribuido intento del Imperio del Sol Naciente (Montalván, 2000). De acuerdo a Montalván, de todos los intentos por llevar a feliz término la negociación canalera, solo cuatro de aquellos proyectos JURÍDICOS pudieron firmarse de los cuales sólo el último de ellos produjo precariamente un beneficio económico.

\subsubsection{PRIMER TRATADO CANALERO 1884.}

Según Montalván, de los cuatro tratados canaleros el primero fue el Zavala - Frelighuysen, negociado a la carrera y con poca seriedad el 01 de diciembre de 1884 cuando paso por Washington el General Joaquín Zavala al dejar la presidencia en viaje oficial a Europa. Resultó que en entrevista apresurada con el Secretario de Estado, le confío Zavala que iba al viejo Continente a explorar la posibilidad de financiar la construcción del canal por Nicaragua. Frelighuysen se apresuró a proponerle un tratado, más para neutralizar el intento negociador del diplomático nicaragüense con los rivales de los norteamericanos que para garantizar el propio compromiso. El tratado no recibió finalmente la aprobación del Senado americano y se extinguió antes de nacer.

\subsubsection{PRIMER INTENTO DE CREAR LA REPUBLICA FEDERAL CENTROAMERICANA} 1887.

Luego sucedería históricamente, uno de los más importantes intentos de reconstrucción de la República Federal Centroamericana el cual surgió por iniciativa del Presidente guatemalteco Manuel Bonilla quien invito a las otras cuatro naciones para que enviaran delegados con propuestas propias a una Asamblea a celebrarse en Guatemala en enero de 1887 el resultado de esta reunión fue la firma del Tratado de Paz Amistad y Comercio, cuando la referida Asamblea finalizó el 16 de febrero de 1887 ya se habían acordado 32 artículos entre los cuales se asentó la no interferencia en los asuntos internos de los Estados (Vásquez, 2011). Como puede observarse era más importante la soberanía que la democracia. El mencionado tratado fue ratificado incondicionalmente por Guatemala, Honduras y Costa Rica, sin embargo, no tuvo mayor trascendencia ya que El Salvador lo ratifico con algunas reservas mientras que el Gobierno conservador de Nicaragua nunca lo ratificó.

\subsubsection{SEGUNDO TRATADO CANALERO 1901 .}

De acuerdo a Álvarez Montalván, el segundo tratado fue el Sánchez - Merry de 1901 firmado por don Fernando Sánchez Ministro de Relaciones Exteriores del Presidente General José Santos Zelaya y el Ministro de los Estados Unidos, en Managua Mr. William

M. Merry. A diferencia del anterior el canal sería manejado íntegramente por los EE.UU, a cambio de lo cual nuestro país recibiría 6 millones de dólares. No obstante el Senado de EE.UU lo rechazó porque para entonces ya había negociaciones para comprar los derechos en la construcción del canal de Panamá (Montalván, 2000). Según un estudio: la autoridad del canal de Panamá, "en la historia del canal de Panamá, el gobierno estadounidense decidió que el lugar adecuado sería Colombia, en su provincia de Panamá. Los Estados Unidos iniciaron negociaciones con el gobierno colombiano, pero este rechazó el proyecto en definitiva (Autoridad del Canal de Panamá, 2013).

Ansiosos por la construcción del canal los Estados Unidos apoyaron un movimiento independentista panameño y así el 03 de noviembre de 1903 Panamá obtuvo su independencia y Estados Unidos adquirió en recompensa por su apoyo, la soberanía del futuro canal, el cual fue inaugurado el 15 de agosto de 1914 . De acuerdo a Díaz Hernán (1998), a comienzos del siglo los Estados Unidos forzó la independencia de Panamá con respecto a Colombia para poder ocupar en forma permanente las inmediaciones del canal interoceánico.

3.2.4. SEGUNDO INTENTO DE CREAR LA REPUBLICA FEDERAL CENTROAMERICANA 1907.

El surgimiento de constantes conflictos en la región entre unos Estados con otros como la última guerra sostenida entre salvadoreños y guatemaltecos en 1906 a falta de un Gobierno General que impusiera el orden 
complicaba las cosas, de manera que muchos intelectuales y escritores centroamericanos como Rubén Darío, Francisco Gaviria y Salvador Mendieta - nicaragüense fundador del Partido de Unidad Centroamericana (PUCA) - se dieron a la tarea de promover la unificación de las repúblicas y el cese al enfrentamiento militar mediante sus publicaciones y escritos.

Al mismo tiempo los Gobiernos de Estados Unidos y México manifestaron su preocupación y se sintieron obligados a buscar algún tipo de solución al problema, en consecuencia invitaron a los Gobiernos centroamericanos a que enviarán delegados a una conferencia que tendría lugar en Washington en 1907 como resultado de la Conferencia se firmó el Tratado General de Paz y Amistad. A pesar de que su primer objetivo fue el mantener la paz en Centroamérica se estuvo muy lejos de obtenerla, porque nuevos enfrentamientos bélicos entre los Estados fueron la respuesta a ese esfuerzo de lograr la paz en la región (Vásquez, 2011).

\subsubsection{TERCER Y CUARTO TRATRADO CANALERO 1913 - 1914.}

De acuerdo a Álvarez Montalván, el tercer tratado, fue negociado y firmado entre don Diego Manuel Chamorro, Ministro de Relaciones Exteriores del Presidente Adolfo Díaz y el Ministro de EE.UU en Managua, Mr. George Weitzel el 08 de febrero de 1913. Pero sucedió que mientras se tramitaba la aprobación del tratado por el Senado, el auspiciador Partido Republicano perdió el poder y ascendió Woodrow Wilson quién designo como su Canciller a Mr. Bryan. Debido a celos políticos, la nueva administración norteamericana repudió todo lo actuado por su antecesor y el tratado quedó sin recibir su aprobación final. Según Montalván, el tratado fue firmado por el General Emiliano Chamorro y el Secretario de Estado William Jennings Bryan el 05 de agosto de 1914 y este concedía a los Estados Unidos el derecho exclusivo y a perpetuidad de construir el canal por Nicaragua lo mismo que el arrendamiento igualmente por tiempo indefinido de bases en el Golfo de Fonseca y el uso del Río San Juan. Nuestro país recibió por esa opción la suma de 3 millones de dólares cuyo uso también fue pactado.

\subsubsection{LA UNIÓN CENTROAMERICANA EN CRISIS CUANDO NICARAGUA FUE OBLIGADA A FIRMAR EL TRATADO CHAMORRO - BRYAN.}

Según Delgado Rojas (2008), ante tal evento la Corte de Justicia Centroamericana (CJC) creada en 1907 cuando conoció la validez del tratado Chamorro - Bryan emitió, sentencia el 09 de marzo de 1917 determinando el retiro de Nicaragua de la Convención y consiguientemente la desaparición de este tribunal. El intenso espíritu cívico existente en la región por las cercanías del centenario de la independencia provoca que después de los fracasos de los pactos de Washington de 1907 surjan movimientos unionistas y entre agosto y septiembre de 1921 se reúnen en la ciudad de Tegucigalpa. Honduras una nueva Asamblea Constituyente, durante sus sesiones ese conclave político discute, redacta y promulga la última Constitución Federal Centroamericana de fugaz validez constituyendo así el último intento de reconstruir la Federación, el aporte más importante de esta Constitución es permitir el sufragio femenino adelantándose en al menos tres décadas a las constituciones políticas de todos los futuros Estados centroamericanos (Rojas, 2009).

De acuerdo a Vicente Vásquez, después de la inventada Federación de 1921 los cinco gobiernos celebraron nuevos pactos en Washington el 07 de febrero de 1923 esta vez sin el patrocinio de México, también se propuso la unión política, pero no existiendo mayoría solamente se firmó el Tratado General de Paz y Amistad, mediante el cual todos los países se comprometieron en no apoyar a ningún gobierno surgido mediante cuartelazos o cualquier otro tipo de derrocamiento.

\section{CONCLUSIONES DE LA PRIMERA ETAPA.}

1. Podemos observar la interferencia sistemática de los Estados Unidos por garantizar sus dominios y sus propios intereses imperialistas en la región centroamericana, estos han contribuido a que los anhelos de unidad, crecimiento, desarrollo y autodeterminación de las Repúblicas se desvanezcan cuando surgen destellos y fuertes deseos de unidad. 
2. El doble juego norteamericano puede identificarse cuando promueven la paz y la unidad entre los centroamericanos y al mismo tiempo organiza y aprueba la continuidad de los golpes de Estado o sus intentos, desde las amenazas desestabilizadoras contra los Gobierno hasta la destitución de Presidentes.

3. Debemos notar también que con estos nuevos pactos de Washington (1923), era la segunda vez que los centroamericanos se iban a discutir asuntos fuera de Centroamérica por los propios centroamericanos. Antecedente que también tiene vigencia actual. Según Álvarez Montalván, esa dependencia mental nos ha entumido para tomar decisiones que nos integren nacional y regionalmente y nos permitan aceptarnos los unos a los otros, sin desconocer intereses divergentes (Montalván, 2000).

4. La primera guerra mundial cambio las relaciones de influencia internacional en América Latina, acelerando el declive de Gran Bretaña como fuerza económica más importante, cuando concluyo la primera guerra mundial el poder y la influencia de Estados Unidos estaba en aumento.

5. Skidmore y Smith, argumentan que la hegemonía ejercida por la potencia norteamericana en la cuenca del Caribe y en Centroamérica se manifiesta en la ocupación militar de Nicaragua entre 1912 - 1925 y 1926 - 1935 (Thomas Skidmore, 1992).

6. Entre 1900 y 1933 las tropas americanas fueron enviadas cuatro veces a Cuba, dos veces a Nicaragua, seis veces a Panamá; y siete veces a Honduras y a la República negra de Haití desde 1915 hasta 1934. Tan grandiosa historia de pillaje y esclavización jugó un papel central en el reparto del mundo por parte de las potencias imperialistas y en la emergencia de una nueva etapa del capitalismo mundial.

\section{ETAPA - II: LA ODECA Y LA GUERRA FRIA.}

Finalmente han sido los mismos centroamericanos los que han buscado la solución de sus problemas, la paz y la unidad de la región, el desarrollo y crecimiento económico. Como consecuencia de esta nueva visión decidieron firmar el 14 de octubre de 1951 la carta de San Salvador que constituye la Organización de Estados Centroamericano (ODECA) creado como un organismo encargado del mantenimiento de la paz y la cooperación en la región y como un mecanismo adecuado para realizar la unión política de los mismos. De acuerdo a Villalta Vizcarra, con la ODECA prácticamente empieza a funcionar la primera

Organización Regional de Centroamérica después que se rompió la República Federal Centroamericana en 1838 (Vizcarra, 2007).

Según Villalta Vizcarra, para los centroamericanos la integración no sólo podía verse como una integración política, sino que también necesitaban de la integración económica por lo que el 13 de diciembre de 1960 en Managua, Nicaragua se firma el Tratado General de Integración Económica Centroamericana y además se crean dos órganos súper importantes que todavía están vigentes que son la Secretaría de Integración Centroamericana (SIECA) que tiene su sede en Guatemala y se crea el Banco Centroamericano de Integración Económica (BCIE) con su sede en Honduras.

Afirma Villalta Vizcarra que con el Tratado General de Integración Económica la región iba prácticamente montada en el mejor carro de la integración, la región empezó a tener lo que se llamaba el Mercado Común Centroamericano (MCCA), empezaron a suprimirse aranceles y ya casi estábamos en la zona de libre comercio (Vizcarra, 2007).

Para entonces los Estados Unidos sostenía una guerra con la Unión Soviética, los Estados Unidos bautizaron a la Unión Soviética como la ideología del mal, mientras ellos encarnaban como era de esperarse, la ideología del bien. Las dos grandes potencias nunca entraron en conflicto directo, de aquí el nombre de "guerra fría" pero si existieron guerras e intervenciones que los involucraban indirectamente, por ejemplo: las guerras de Corea, Vietnam, Afganistán y Nicaragua. El corazón de la guerra fría consistió en los intentos soviéticos por expandir su influencia comunista y las consecuentes acciones estadounidenses para frenarlos.

\subsection{EL LEGADO DE NICARAGUA Y AGUSTO C. SANDINO.}

Mientras tanto la semilla de Sandino había germinado en Latinoamérica y especialmente en Nicaragua con diversos resultados en la juventud que vio caer a Sandino vilmente asesinado. Somoza García al planear el asesinato de Augusto C. Sandino, eliminó a su competidor despejando su camino hacia la dictadura dinástica 
que duró 43 años, ingenuamente Anastasio Somoza Debayle en 1979 especuló hasta el último momento que el Gobierno norteamericano vendría con sus Globe's Masters a rescatarle del comunismo,al final solo y abandonado por su padrino foráneo, abandonó el poder huyendo en un helicóptero al extranjero (Sequeira, 2003).

Una vez derrocada la dictadura Somocista en julio de 1979. De acuerdo a Villalta Vizcarra aparecen nuevos conflictos internos en la región sobre todo en Nicaragua, El Salvador y Guatemala, pero según Herdocia Sacasa nuevamente los Estados Unidos hacían su intervención esta vez apoyando hasta en misiones paramilitares a los grupos armados con presencia en la región centroamericana, entonces era necesario pacificar la región, democratizar la región porque había mucha crisis entre la misma población civil, conflictos entre los Estados y era, muy necesario encontrar una salida negociada a esos conflictos y es cuando aparecen los procesos de Contadora (1983, 1985 y 1986) El Grupo de Apoyo (1985), Esquipulas I (1986) y Esquipulas II (1987) (Herdocia, 2003).

\subsection{PRINCIPALES INICIATIVAS JURÍDICAS PARA ESTABLECER LA PAZ Y LA DEMOCRACIA EN CENTROAMERICA.}

\subsubsection{EL PROCESO DE CONTADORA.}

En un estudio sobre Integración Regional y Democracia especial atención a Centroamérica podemos describir los diferentes procesos e iniciativas jurídicas que fueron impulsadas para establecer la paz y la democracia en la región centroamericana. CONTADORA (Isla de la República Panameña) es un proceso donde la comunidad internacional trata de ayudar a los países centroamericanos a tener una solución pacífica de controversias en base al dialogo, los países que la integraban eran México, Venezuela, Colombia y Panamá (Herdocia, 2003).

Esa acta se llamó: "El Proyecto de Acta de Contadora para la Paz y la Cooperación de Centroamérica" estas actas no se firmaron, pero existen. Es necesario recordar que no se firman las tres actas de CONTADORA porque indudablemente había un gran peso de Estados Unidos en esta decisión de los países centroamericanos de no suscribir el acta de CONTADORA.

\subsubsection{EL GRUPO DE APOYO.}

De acuerdo a Herdocia Sacasa, el Grupo de Apoyo estaba formado por Perú, Brasil, Argentina y Uruguay, lo importante de este proceso en Centroamérica es que éste es el antecedente del Grupo de Río, un foro político muy importante que se reúne para resolver problemas políticos del Continente Americano. Aquí se vislumbran nuevos modos de vivir la seguridad en la región y de vivir de manera democrática.

Aquí viene la parte que muy poca gente conoce y es que Centroamérica hace un aporte extraordinario a la identidad cultural Latinoamericana al mediar en la crisis regional los diferentes grupos fueron construyendo un conjunto de valores compartidos, esto no solo implica la apertura de la sociedad al pluralismo político, sino además el cese de políticas intervencionistas y esta interdependencia iba a permitir la solución de los conflictos y esto fue gracias a Nicaragua (Herdocia, 2003).

\subsubsection{LOS PROCESOS DE ESQUIPULAS.}

Posteriormente surgen los procesos de Esquipulas I del 25 de mayo de 1986 y Esquipulas II del 06 y 07 de agosto de 1987 los que permitieron la incorporación de las antiguas fuerzas armadas irregulares a los procesos políticos permitiendo la democratización y permitiendo también enfrentar la injerencia de los Estados Unidos, los acuerdos de Esquipulas vinieron a frenar ese tipo de injerencia.

Según Herdocia Sacasa, estos fueron los acuerdos más importantes jamás suscritos por Centroamérica después de su Acta de Independencia. Esquipulas II de 1987 es un procedimiento para establecer la paz firme y duradera en Centroamérica, es un antes y un después en la historia regional y es imposible explicar todos los procesos de paz y reconciliación sin la voluntad del Gobierno Sandinista Revolucionario en esa muy difícil primera segunda etapa (Herdocia, 2003).

De acuerdo a Cruz Sequeira, así se explica el fin de la guerra fría en Latinoamérica, las potencias que ayudaban, abandonaron a sus respectivos protegidos. En efecto enterado el Congreso norteamericano del 
inminente desmoronamiento del Imperio Soviético negó en 1987 aprobar fondos para el financiamiento de la resistencia nicaragüense. A su vez el viceministro de la Unión de Repúblicas Socialistas Soviéticas (URRS) visitó Managua y notificó al Frente Sandinista de Liberación Nacional (FSLN) que debía arreglarse con su oposición porque ellos ya lo habían hecho con los EE.UU (Sequeira, 2003).

No hay que olvidar nunca que la paz y la democracia llegan a Centroamérica en la década de los 90 desde un vehículo no de negociaciones bilaterales sino desde un vehículo de concepción general, la pacificación y la democratización de la región solamente se pudo vislumbrar a través del proceso de Integración Regional. Después de la reunión de Presidentes en 1990 en Puntarenas, Costa Rica y recién finalizado el conflicto en Nicaragua, pero todavía pendiente en El Salvador y Guatemala se proclama Centroamérica como región de paz, libertad, democracia y desarrollo (Herdocia, 2003).

\subsection{EL SISTEMA DE LA INTEGRACIÓN CENTROAMERICANA.}

El Sistema de la Integración Centroamericana (SICA) nace como resultado de la superación de los conflictos armados en la región centroamericana. Según Villalta Vizcarra, el Protocolo de Tegucigalpa firmado el 13 de diciembre de 1991 es el que crea el Sistema de la Integración Centroamericana (SICA) y tiene como propósito promover y consolidar la democracia representativa dentro de los principios de no intervención en los asuntos internos y se establece sanción de suspensión de la organización por la violación a los principios democráticos (Vizcarra, 2007).

Por lo tanto, con la firma del Protocolo de Tegucigalpa se da un paso gigantesco que ya los centroamericanos en cierta medida habían vislumbrado en los acuerdos de Washington de 1907 y 1923 cuando indicaron que no iban a reconocer a Gobiernos surgidos de golpes de Estados suspendiendo el proceso democrático.

\section{CONCLUSIONES DE LA SEGUNDA ETAPA.}

1. Las justificaciones para intervenir en la región centroamericana de los norteamericanos son múltiples y variadas desde las posibilidades de construcción del canal interoceánico hasta la presencia del odiado comunismo han sido motivos suficientes para desplegar su poderoso brazo militar en algunos casos y sus misiones paramilitares y diplomáticas en otros.

2. Lo más importante en esta segunda etapa fue que Centroamérica volvía a recuperar la cláusula Tobar de (1907) del Ministro ecuatoriano Carlo R. Tobar, cuando dijo que los Estados centroamericanos con el fiel apego a la carta democrática interamericana no reconocerían a ningún Estado surgido de la alteración al orden constitucional (Herdocia, 2003).

3. Cuando surge la doctrina Tobar surge un movimiento en el sistema interamericano dirigido a contener la intromisión de los Estados y es cuando surge la doctrina de Genaro Estrada Secretario de Relaciones Exteriores mexicano, el 27 de septiembre de 1930, que es todo lo contrario a la doctrina Tobar. La doctrina Estrada es: nadie debe meterse a calificar si un Gobierno es o no democrático, eso es un asunto interno de los Estados y a él le corresponde decidir su propio destino.

4. Otro aspecto importante según Herdocia Sacasa, es que en Centroamérica ya existe una acción colectiva solidaria pro - democracia y que la amenaza al proceso democrático en cualquiera de los países centroamericanos constituye también una amenaza a los demás miembros, haciendo extensiva aquella primicia de asistencia recíproca que establece que toda agresión a un Estado se extiende a los demás Estados.

5. Podemos descifrar fácilmente la doble moral de la política exterior norteamericana en la región centroamericana cuando el subsecretario de Estado para Asuntos Interamericanos Alexander F. Watson anunció que la Administración Demócrata del Presidente Bill Clinton tenía un mensaje muy claro para los nicaragüenses: "Busquen entre ustedes un acuerdo nacional por medio del dialogo no busquen las respuestas a sus problemas en Washington".

6. Guardando las distancias con el gran teatro de la política mundial en el caso de Nicaragua el gobierno de Estados Unidos a partir del 9/11 no sólo ha expresado su deseo por una sociedad política moderna en nuestro país, es decir, libre de caudillos sobre todo del caudillo sandinista, 
7. En el caso del Partido Liberal Constitucionalista (PLC), las reservas de los norteamericanos no son con sus militantes, sino que con el ex presidente Arnoldo Alemán, y en el caso del Frente Sandinista de Liberación Nacional (FSLN), han tenido que reconocer que sus reservas se limitan a la "Primera Generación" de sandinistas, con el Presidente Ortega a la cabeza.

8. Así los Estados Unidos dentro del nuevo espíritu de su internacionalismo militante, decidieron micro administrar los asuntos nicaragüenses al menos los del Gobierno del Ingeniero Enrique Bolaños y del Partido Liberal. El objetivo es sencillo, por medio de su embajada en Managua como en tiempos de otrora, los Estados Unidos pretenden alinear a los liberales en un solo "Polo Democrático" excluyendo al ex presidente Alemán y al FSLN del Presidente Daniel Ortega del equilibrio nacional. Este ha sido el esfuerzo de los Estados Unidos por más de dos años, y la última expresión de esta voluntad fue la misión especial, y aparentemente infructuosa del Embajador Oliver Garza a Nicaragua durante el mes de agosto del 2005 (Sequeira, 2003).

\section{Referencias Bibliográficas}

Autoridad del Canal de Panamá. (2013). Informe Anual del Canal de Panamá. Panamá: Universitaria.

Cincotta, H. (1994). An outline of American history. Estados Unidos: Agencia de Información.

Díaz, E. 2013. Sobre el denominado "Mediterraneo americano" In XV Encuentro de Latinoamericanistas Españoles. Trama Editorial, 1337 - 1350.

Herdocia, M. (2003). Integración y modelo de seguridad democrática en Centroamérica: su influencia dentro de la OEA. Revista de Derecho, 185 - 214.

McDougall, W. A. (1997). Promised land, crusader state:the American encounter with the world since 1776. United States: Houghton Mifflin Company.

Montalván, E. Á. (2000). Cultura Politica Nicaraguense. Managua: HISPAMER.

Rojas, J. D. (2009). Construcciones Supranacionales e integración regional latinoamericana. San José. Costa Rica: UCR. Sequeira, A. C. (2003). La república conservadora de Nicaragua 1858 - 1893.

Stephanson, A. (1996). Manifest Destiny: American expansion and the empire of right. New York: Hill and Wang. Thomas Skidmore, P. H. (11 de Abril de 1992). Modern Latin America. New York: Oxford University.

Vásquez, G. V. (2011). Nacimiento y ocaso de la Federación de Centro América:entre la realidad y el deseo. España: Revista Complutense de Historia de América.

Vizcarra, A. E. (2007). LA CARTA DEMOCRÁTICA INTERAMERICANA Y EL TRATADO MARCO DE SEGURIDAD DEMOCRÁTICA EN CENTROAMERICA. Miembro del Comité Jurídico Interamericano. Recuperado el 12 de Abril de 2016, http://www.fes- seguridadregional.org/images/stories/ docs/5182-001_g.pdf

Weeks, E. W. (1997). Building the continental empire:American Expansion from the Revolution to the Civil War. Chicago: Ivan R. Lee. Inc. 